\title{
The Effects of Extraneous Load on the Relationship Between Self-Regulated Effort and Germane Load Within an E-Learning Environment
}

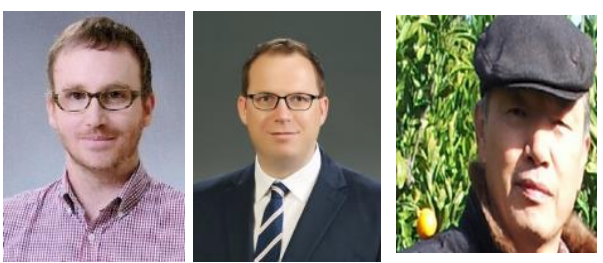

Dr. Christopher Lange ${ }^{1}$, Dr. Jamie Costley², and Dr. Seung-lock Han ${ }^{3}$

${ }^{1}$ Joongbu University, ${ }^{2}$ Kongju National University, South Korea, ${ }^{3}$ Kongju National University, College of Education

\begin{abstract}
Online instructors need to avoid unclear and confusing explanations of content, which can reduce the quality of learning. Extraneous load is reflective of poor instruction, in that it directs student effort towards processing information that does not contribute to learning. However, students may be able to manage poor instruction through effort regulation. Students who show high levels of effort have been shown to overcome poor instruction in some cases. This study analyzed survey responses from South Korean university students studying online $(n=1,575)$ to examine the relationship between self-regulated effort and germane load within varying extraneous load conditions. The experimental design separated extraneous load responses into three conditions (low, medium, high). Within each extraneous load condition, self-regulated effort responses were also separated (low, medium, high). The results showed that as extraneous load increased, self-regulated effort had a weaker relationship with germane load. It was also found that the use of effort regulation is effective only when dealing with low and mid-level extraneous load situations and that use of such strategies within high extraneous load situations was not effective. These results show the importance of improving instruction to reduce extraneous cognitive load, in that, not even high levels of effort can overcome poor quality instruction.
\end{abstract}

Keywords: e-learning, cognitive load, distance learning, extraneous load, germane load, online learning, self-regulated effort, self-regulated learning

\section{Introduction}

Because of the physical separation between the learner and the classroom, distance learning magnifies the need for not only effective instruction, but also for specific learning strategies that help manage content delivered by instructors. As a part of distance learning, e-learning is defined as instruction 
delivered via computer network connectivity, which is designed to promote learning through the presentation of content represented by various electronic sources (Mayer, 2003). Because students are faced with the challenge of selecting relevant content delivered to them through multiple sources of information, e-learning requires a degree of autonomy than offline learning (Gerjets \& Scheiter, 2003; Mayer, 2014). Cognitive load may be greater if content used in e-learning is not clearly presented (Gerjets \& Scheiter, 2003; Kalyuga, Chandler, \& Sweller, 1999). Instructors need to deliver content in a way that will not overload the cognitive processing of the learners. However, if cognitive overload does occur, students may benefit from effort regulation strategies, which have been found to be beneficial within online learning environments (Puzziferro, 2008).

Cognitive load represents a process of information transfer by learners from their working memory to their long- term memory (De Jong, 2010; Sweller, 2005; Sweller, Van Merriënboer, \& Paas, 1998). Specifically, it refers to the fact that because there is limited cognitive capacity in the working memory, learning can be negatively affected if information that needs to be processed exceeds that capacity (De Jong, 2010). Cognitive Load Theory addresses cognitive processes through the examination of the following three elements: intrinsic load, extraneous load, and germane load (De Jong, 2010; Sweller, 2005; Sweller, Van Merriënboer, \& Paas, 1998). Intrinsic load refers to the complexity of the material coupled with prior knowledge of the learners (Sweller \& Chandler, 1994). Although there is some research that shows ways in which to minimize intrinsic load through strategic the use of specific materials, it is more commonly accepted that intrinsic load cannot be dramatically affected by instructional strategies (Cierniak, Scheiter, \& Gerjets, 2009; Shadiev, Hwang, Huang, \& Liu, 2015). Extraneous load reflects poor instructional delivery, which causes unnecessary processing within short-term memory to a point where student learning is negatively affected (Cierniak et al., 2009; Leppink, Paas, Van der Vleuten, Schmeck, Opfermann, van Gog, Paas, \& Leutner, D, 2015; Van Gog, \& Van Merriënboer, 2013). As it is strongly related to poor instructional quality, extraneous load can be altered through instructional intervention (Sweller, 2005). Instruction may contribute to higher levels of extraneous load if it includes the delivery of content in an unclear fashion (Leppink et al., 2013). Germane load is the only element of cognitive load theory where high levels are considered positive for the learning experience (Cierniak et al., 2009). More specifically, germane load directly contributes to learning and represents how well the students understand the content (Homer, Plass, \& Blake, 2008; Sweller et al., 1998). Although germane load levels are affected by the amount of effort exerted by learners, there is disagreement about the process by which effort affects germane load. An ideal learning environment is one that promotes high levels of germane load through the reduction of extraneous load (Cierniak et al., 2009; Sweller et al., 1998).

Self-regulated learning is defined as the ability to show independent and proactive engagement through self-motivation and behavioral techniques used to accomplish goals (Zimmerman, 2008). Self-regulated learners are persistent when confronted with difficult tasks, and are able to keep away from behaviors that distract them from the learning process (Dabbagh \& Kitsantas, 2012). Furthermore, self-regulated learners believe that taking on challenging tasks, developing a deep understanding of content, and using more effort will enhance their learning and ultimately help them succeed in their coursework (Perry, Phillips, \& Hutchinson, 2006). Zimmerman and Pons (1986) provide a list of strategies often used by self-regulated learners in order to persist in challenging tasks, including goal-setting and planning, self-evaluating, self-monitoring, organizing and transforming, seeking assistance from peers, and seeking assistance from instructors. The Motivational Strategies 
for Learning Questionnaire (MSLQ) was developed to reflect the way learners use their beliefs and cognition to formulate motivational learning strategies (Pintrich, Smith, Garcia, \& McKeachie, 1991). Although many of the MSLQ strategies address the effort put forward by students to succeed, the selfregulated effort strategy from MSLQ is useful to examine a more general sense of effort given when students face challenging content. Self-regulated effort is defined as the amount of commitment a student dedicates to managing specific instructional tasks that are perceived as difficult to complete (Pintrich et al., 1991). The use of such effort is believed to act as an agent for overcoming ineffective instruction. For example, it has been said that self-regulated learners put in effort and find ways to succeed, even when presented with incomprehensible material, inadequate study conditions, and confusing instruction (Gerjets, Scheiter, \& Tack, 2000; Leutner \& Sumfleth, 2009; Moos, 2013; Zimmerman, 1990).

\section{Theoretical Background}

\section{Relationship Between Extraneous Load and Germane Load}

Both extraneous load and germane load are associated with effort put forth by learners. While effort associated with extraneous load is a result of poor instructional practices that cause unnecessary processing, effort associated with germane load contributes to processes that are reflective of learning (Kirschner, 2002). When instructional design effectively minimizes extraneous load, learners can effectively construct schema by engaging in relevant activities that contribute to learning, further enhancing levels of germane load (De Jong, 2010; Sweller et al., 1998). Within e-learning multimedia environments specifically, extraneous load can negatively affect cognitive processes that lead to learning. Mayer (2014) showed that if extraneous materials are added to parts of a lecture that contains essential information, students will have lower levels of comprehension. Additionally, splitting learners' attention between multiple sources of information presented simultaneously has been shown to create extraneous processing, which negatively affects learning (Mayer \& Moreno, 2003; Moreno, Mayer, Spires, \& Lester, 2001). Furthermore, reducing extraneous load has been shown to be beneficial to learning. The redundancy effect shows that redundant information introduced to the learning environment leads to extraneous processing. Mayer, Heiser, and Lonn (2001) set two experimental conditions; one where narration was simultaneously accompanied by onscreen text displaying the exact same words being used in the narration and one where that on-screen text was removed during the narration. The findings showed that the reduction of the extraneous onscreen text led to better understanding of the online multimedia presentation.

\section{Relationship Between Extraneous Load and Self-Regulated Effort}

Students' use of specific strategies is often determined by the learning environment itself. Selfregulated learning strategies such as effort regulation are not static, but contextually bound through the type of instruction delivered within specific learning environments (Duncan \& McKeachie, 2005). The quality of instruction represents a specific environmental factor that may influence students' effort through the use of self-regulated learning strategies. The amount of effort students employ is often related to their perceptions of how effective that effort would be, given specific circumstances within their environment (Paas, Tuovinen, van Merriënboer, \& Darabi, 2005). In other words, if instruction contains elements that lead to extraneous processing, learners may perceive those 
elements as unnecessary for success, therefore they may reduce their effort. Additionally, the cognitive processing associated with extraneous load is further compounded by the cognitive processing required for self-regulation, which may lead to a reduction of effort, given the limited processing power afforded to individuals at a specific time (Saw, 2011). Such a reduction of effort within high extraneous load environments has been empirically examined. For example, extraneous questioning provided by instructors within a hypermedia environment has been shown to have a negative impact on the use of self-regulated learning strategies, including re-reading, taking notes, and coordinating informational sources (Moos, 2013). Although poor instruction has been shown to negatively affect levels of student effort, it should be noted that not all students learn the same way under the same conditions. Self-regulation still occurs within high extraneous load environments, as some learners attempt to compensate for poor instruction in order to succeed. Gerjets and Scheiter (2003) acknowledge that the specific constraints associated with ineffective instruction do affect learning strategies, but that different types of learners who receive the same instruction will still vary in regards to their use of such strategies. Furthermore, different patterns of learning are evident as a result of variations of strategies that students will use within the same learning environment (Gerjets et al., 2000; Gerjets \& Scheiter, 2003).

\section{Relationship Between Self-Regulated Effort and Germane Load}

Germane load is a product of the motivational effort used to construct schema, resulting in a positive learning experience (De Jong, 2010). Reflective of this notion, persistence when encountering boring or difficult instruction has been linked to academic achievement (Komarraju \& Nadler, 2013; Pintrich et al., 1991). The effort associated with self-regulation, specifically, is a critical aspect of distance learning, as it represents a major determinate of what is learned within those environments (Saw, 2011). For example, student self-regulation through better planning and effort within multimedia elearning environments has been shown to lead to higher levels of learning (Moos, 2013; Moos \& Azevedo, 2008). The use of self-regulation involving memorization, elaboration, and organization has been shown to positively affect learner achievement in e-learning environments (Shih, Ingebritsen, Pleasants, Flickinger, \& Brown, 1998). Furthermore, self-regulated effort was found to be associated with an increase in academic performance within a college e-learning course (Puzziferro, 2008).

\section{Relationship Between Specific Levels of Self-Regulated Effort and Germane Load Within Varying Extraneous Load Conditions}

Various studies have examined the effects that different levels of self-regulation have on learning within both high and low extraneous load e-learning environments. Within low extraneous load environments, studies have shown that self-regulation strategies increase and higher levels of learning occur as a result (Moos \& Azevedo, 2008; Kramarski \& Mizrachi, 2006). Within high extraneous load environments, research has shown that instruction that imposes extraneous load on the learners is associated with less usage of self-regulated learning strategies, and that low levels of self-regulation negatively affect learning (Moos, 2013). Although these studies provide insight into the processes occurring within both high and low extraneous load environments, they are limited in their scope. They are predicated on the fact that low levels of extraneous load increase self-regulated learning and high levels of extraneous load decrease self-regulated learning, essentially disregarding the effect that low self-regulation has on learning within low extraneous load environments and the effect that high self-regulation has on learning within high extraneous load environments. In order to get a complete picture, low self-regulation within low extraneous load environments and high self-regulation within 
high extraneous load environments need to be examined.

Gerjets and Scheiter (2003) contend that students learn in different ways, and that student strategies will still vary regardless of the learning environment. Therefore, unlike other research, they examined low self-regulation within low extraneous load environments and high self-regulation within high extraneous load environments. Within a low extraneous load online environment, Gerjets et al. (2000) looked at both low and high levels of self-controlled learning strategies and found that students who displayed less effort through low levels of processing strategies suffered from lower performance levels than those that utilized more effort through higher levels of processing strategies. Gerjets et al. (2000) also showed that when extraneous load was increased through the reduction of learning time, those that used higher levels learner control processing strategies were able to overcome the ineffective instruction to a point where learning was not negatively affected. This shows that selfregulation can be used to overcome ineffective instruction that imposes time constraints, as students apparently were able to apply their effort to aspects of the environment that contribute to learning and ignore irrelevant information unnecessary for learning (De Jong, 2010).

\section{The Current Study}

Online learning deserves attention from a cognitive load perspective, as delivery of instruction often requires a level of strategic effort to deal with aspects of cognitive load that may be imposed on the learner. Research has shown that multimedia online environments are at a greater risk of inducing extraneous load, and that extraneous load is negatively correlated with germane load (Gerjets \& Scheiter, 2003; Kalyuga et al., 1999). Additionally, it has generally been found that extraneous load occurring in e-learning leads to lower levels of effort (Moos, 2013; Moos \& Azevedo, 2008; Kramarski \& Mizrachi, 2006), although higher levels of effort still occur within high extraneous load environments as some learners attempt to compensate for poor instruction (Gerjets et al., 2000; Gerjets \& Scheiter, 2003). Use of self-regulation within e-learning has proven to be useful in overcoming poor instruction, as high levels of self-regulation strategies have been shown to lead to better performance (Moos, 2013; Moos \& Azevedo, 2008; Puzziferro, 2008; Shih et al., 1998). Finally, past research has postulated and shown evidence that students who show higher levels of effort can overcome ineffective instruction in order to succeed (Gerjets et al., 2000; Leutner \& Sumfleth, 2009; Moos, 2011; Moos, 2103; Zimmerman, 1990).

Although it has been shown that both instruction and learning strategies can affect outcomes within elearning, it is useful to examine the relationship between unclear instruction, represented by extraneous load, and learning, represented by germane load. This is important in order to see if student effort can overcome unclear instruction within university online learning environments. To investigate this, the current study analyzed survey responses from university students in South Korea and separated their extraneous load responses into three conditions (high, medium, low). Within those conditions, self-regulated effort was also divided into three levels (high, medium, low) to see the relationship that different levels of self-regulated effort have with germane load within a high, medium, and low extraneous load condition.

\section{Research Questions}


1. Is there a statistically significant relationship between extraneous load and germane load?

2. Is there a statistically significant difference between extraneous load and self-regulated effort?

3. Is there a statistically significant difference between self-regulated effort and germane load?

4. Is there a difference in germane load means between the self-regulated effort groups in the low extraneous load condition?

5. Is there a difference in germane load means between the self-regulated effort groups in the moderate extraneous load condition?

6. Is there a difference in germane load means between the self-regulated effort groups in the high extraneous load condition?

\section{Methods}

\section{Context and Participants}

This study surveyed a group of students in South Korea who received instruction within Open Cyber University (OCU) online courses. This institution first started delivering online instruction in the fall semester of 1998 (Jung \& Rha, 2001). The OCU is associated with 23 traditional brick-and-mortar universities who provide both funding and administrative oversight (Jung, 2000). The OCU offers approximately 400 different classes, and 120,000 students per semester are enrolled (Han, 2012). The 23 member universities also provide the instructors and course titles for instruction delivered in the OCU (Jung \& Rha, 2001). While there are a small amount of courses that include face-to-face tutoring and offline assessment, most instruction in the OCU occurs through web-based lectures and quizzes (Jung \& Rha, 2001). The membership universities provide the content and design for the online courses (Jung \& Rha, 2001). Online courses that make up the OCU generally consist of instructorcentered web-based instruction with very limited or no human interaction among its participants (Jung \& Rha, 2001).

The participants in this study filled out a Google docs form in the spring semester of 2016. 1801 students filled out the survey; however 226 of those surveys were removed from the analysis because they were incomplete, or for the purpose of making the nine differing groups used in this study equal in size. This research procedure is similar to that of McManus (2000) where low, medium, and high self-regulated learning groups were compared. Of the 1575 students who completed surveys, 756 (48\%) were male and 819 (52\%) were female. The youngest participant in this study was 15 , and the oldest was 63 . The average age of the study participants was 23.5, with a standard deviation of 3.2. Other studies involving online learning environments have found similar distributions of age and gender (Suh \& Kim, 2013). The participants responded to the survey based on specific OCU courses they took during the semester. The courses were categorized as follows, with the percentage of participants taking each course-type in parentheses: liberal arts (33\%), social science (17\%), technology (15\%), lifestyle and health (12\%), management and business (8\%), foreign language (7\%), natural science 
(6\%), and design (2\%). The distribution of subject areas is similar to the general distribution found in the OCU (Kobayashi \& Kim, 2010).

\section{Research Procedures and Data Collection}

This study began with qualitative interviews of 10 students who had participated in OCU classes in the fall semester of 2015. These interviews were focused on learner-to-learner interaction, the students' general perceptions of the OCU, and their learning experiences. These interviews helped define and generate questions for a broad survey covering all aspects of the OCU that were given to 92 students in January of 2016. This survey was general but focused around the direct instruction the learners received with questions regarding lesson quality, watching lectures, academic honesty, learner-tolearner interaction, instructor interaction, and learning materials. From this survey, it was evident that the learning experience of students was variable in terms of the video lectures themselves. However, all participants interviewed expressed that there was no significant interaction or activities to supplement the video lectures. Because of this, a decision was made to generate a more specific survey that focused on aspects within the video lectures and distribute it to a larger sample of OCU students.

The survey used in this research was initially written in English, and then translated into Korean. An expert in both the OCU and online learning checked the translation and found it to be accurate. A link to the survey was sent to the OCU's central administrative offices, where it was checked for errors or issues. The link along with a message inviting students to participate in the research was then posted on the OCU's main information board, and was active from April 20th to May 20th, 2016.

\section{Instruments}

To create the extraneous load and germane load scale, four items for germane load and seven items for extraneous load were used and measured using a Likert-type scale ranging from 0 to 10 , with 0 being "strongly disagree" and 10 being "strongly agree." The justification for using the o to 10 Likerttype scale for this study is due to the fact that the original items were designed with this scale, and to ensure reliability, the same scale was used in this study. To measure germane load, four items from Leppink et al.'s (2013) paper titled "The development of an instrument for measuring cognitive load" were adapted. The four items used to measure germane load in the present study were: The lecture really enhanced my understanding of the topic, the lecture really enhanced my knowledge and understanding of the of the class subject, the lecture really enhanced my understanding of the concepts associated with the class subject, and the lecture really enhanced my understanding of concepts and definitions. Cronbach's Alpha was measured and found to be .961, which is acceptable for this research. The three items measuring extraneous load were also adapted from Leppink et al. (2013). The three items used in this study to measure extraneous load were: The explanations during the lecture were very unclear; the explanations were, in terms of learning, very ineffective; and the explanations were full of unclear language. The Cronbach's Alpha was calculated and found to be .946 , which is also acceptable for this type of research.

The measurements used to calculate self-regulated effort come from the Motivated Strategies for Learning Questionnaire (MSLQ) that is used to assess college students' motivational orientations and their use of different learning strategies (Pintrich et al. 1991). The Likert-type scale used for these items was set at a range from o to 10 , with o representing "disagree" and 10 representing "agree." 
Although the MSLQ was originally designed to be set at a 7-point Likert-type scale, the current study set it at $\mathrm{o}$ to 10 to ensure consistency with the range throughout the entire survey. This was done because the main constructs used in this study are the cognitive load constructs, which are set at a o to 10 range. Justification for altering the range is provided by various studies (Ergul, 2004; Nie, Lau, \& Liau, 2011; Simon, Aulls, Dedic, Hubbard, \& Hall, 2015). From the MSLQ, the following four items were adapted for use in this study to measure self-regulated effort: I often lose focus when I study so I quit before I finish what I planned to do (reversed); I work to do well at school even if I get confused; when coursework is unclear, I give up or only study the easy parts (reversed); and even when study materials are complex, I manage to keep working until I finish. The Cronbach's Alpha was calculated and found to be .727 , which is acceptable for this type of research.

\section{Experimental Design}

To create the different groups for examining the relationships between self-regulated effort and germane load within varying extraneous load conditions, the study participants were separated into three different groups (low, medium, high). This means that there were a total of nine separate conditions that were compared as the main part of this study. The 1575 participants' levels of extraneous load were calculated and ordered. The 525 subjects with the highest levels of extraneous load represented the "high" extraneous load group, the next 525 subjects represented the "medium" extraneous load group, and the lowest 525 subjects represented the "low" extraneous load group. This method of dividing participants is similar to the method used in Chang and Yang's (2009) cognitive load study. The grouping procedure for self-regulated effort also included dividing the overall sample into three groups, with 525 participants with the highest levels of self-regulated effort being the "high" group, the next 525 participants being the "medium" group, and the 525 participants with the lowest levels of self-regulated effort being the "low" group. This method of dividing subjects into groups based on their relative levels of self-regulated effort is the same method used in McManus (2000) and Wang (2011).

\section{Results}

The first step in understanding the relationships between the three main variables was to look at their direct correlations. This was done to show the degree to which the main variables were correlated with each other, and to see if those relationships were statistically significant. Table 1 shows that all of the variables have a statistically significant positive or negative relationship. Extraneous load is negatively correlated with both self-regulated effort (-.261) and germane load (-.602). Also, self-regulated effort is positively correlated with germane load (.437). All of these correlations were significant at the .01 level using Spearman's correlation coefficient. All told, the direction of these correlations suggest that extraneous load leads to less germane load and less self-regulated effort, while self-regulated effort leads to higher levels of germane load.

Table 1

Correlations Between Extraneous Load, Self-Regulated Effort, and Germane Load ( $=1575)$ 


\begin{tabular}{|l|l|l|l|l|}
\hline & & 1 & 2 & 3 \\
\hline 1 & Extraneous Load & 1 & & \\
\hline 2 & Self-Regulated Effort & $-.261^{* *}$ & 1 & \\
\hline 3 & Germane Load & & & \\
\hline
\end{tabular}

** Correlation is significant at the .o1 level

The first stage of examining the relationships between the variables was to separate them into different groups to be used for analysis in this study. First, the overall mean levels for the three variables were examined Table 2 shows that extraneous load had a mean value of 3.48, self-regulated effort had a mean value of 6.85 , and germane load had a mean value of 6.29.

Table 2

Means for Extraneous Load, Self-Regulated Effort, and Germane Load ( $N=1575)$

\begin{tabular}{|l|l|c|c|c|c|}
\hline & & Min & Max & Mean & SD \\
\hline 1 & Extraneous Load & 0.00 & 10.00 & 3.48 & 2.56 \\
\hline 2 & Self-Regulated Effort & 3.00 & 10.00 & 6.85 & 1.64 \\
\hline 3 & Germane Load & 0.00 & 10.00 & 6.29 & 2.78 \\
\hline
\end{tabular}

After the three main variable descriptive statistics were examined, the participants were split into three groups based on their levels of perceived extraneous load. These groups were low, medium, and high. Table 3 shows that the low extraneous load group had a mean extraneous load of 0.62 , the medium extraneous load group had a mean extraneous load of 3.59, and the high extraneous load group had a mean extraneous load of 6.20. After the high, medium, and low extraneous load groups were created, the participants were also divided based on their self-regulated effort scores. Table 3 shows that there were three self-regulated effort groups created within each extraneous load group, with 175 participants in each. In the low extraneous load group, the low effort group had an effort mean of 5.605, the medium group had a mean of 7.604, and the high effort group had mean of 9.472. The medium extraneous load group also had three groups with the low effort group having an effort mean of 4.930, the medium group having a mean of 6.164, and the high group having an effort mean of 8.044. In the high extraneous load condition, the low effort group had an effort mean of 5.080, the medium group had a mean of 6.235, and the high group had a mean of 8.354.

Table 3 


\begin{tabular}{|l|c|c|c|c|c|c|c|}
\hline & $n$ & EL & SD & & $N$ & Effort & SD \\
& & Mean & & & & Mean & \\
\hline Low EL & 525 & 0.62 & 0.72 & Low Effort & 175 & 5.60 & 0.65 \\
& & & & Medium Effort & 175 & 7.60 & 0.62 \\
& & & & High Effort & 175 & 9.47 & 0.65 \\
\hline Medium & 525 & \multirow{2}{*}{3.60} & \multirow{2}{*}{0.99} & Low Effort & 175 & 4.93 & 0.31 \\
& & & & Medium Effort & 175 & 6.16 & 0.48 \\
\hline High EL & 525 & \multirow{2}{*}{6.20} & \multirow{2}{*}{1.61} & Low Effort & 175 & 5.08 & 0.29 \\
& & & & Migh Effort & 175 & 8.04 & 0.85 \\
& & & & Medium Effort & 175 & 6.24 & 0.42 \\
& & & & High Effort & 175 & 8.35 & 1.03 \\
\hline
\end{tabular}

This created nine potential combined conditions as can be seen in Table 3 above and Table 4 below. Next, using the nine different combined groups, the germane load means for each of the conditions were examined (Table 4). The condition with the highest germane load mean was the low extraneous load, high effort group with a germane load mean of 9.328 and the condition with the lowest germane load mean was the high extraneous load, low effort group with a mean germane load of 4.815. The means for the other conditions can be seen in Table 4 .

Table 4

Mean Germane Load by Extraneous Load and Effort Groups

\begin{tabular}{|c|l|l|l|l|}
\hline EL groups & Effort groups & $N$ & $\begin{array}{l}\text { Germane Load } \\
\text { Mean }\end{array}$ & SD \\
\hline Low EL & High Effort & 175 & 9.33 & 1.22 \\
\hline Low EL & Medium Effort & 175 & 8.14 & 1.42 \\
\hline Low EL & Low Effort & 175 & 6.43 & 2.51 \\
\hline
\end{tabular}




\begin{tabular}{|c|c|c|c|c|}
\hline \multicolumn{2}{|c|}{ Total for Low EL } & 525 & 7.99 & 2.16 \\
\hline Medium EL & High Effort & 175 & 6.84 & 1.64 \\
\hline Medium EL & Medium Effort & 175 & 5.80 & 1.28 \\
\hline Medium EL & Low Effort & 175 & 5.13 & 0.97 \\
\hline \multicolumn{2}{|c|}{ Total for Medium EL } & 525 & 5.93 & 1.50 \\
\hline High EL & High Effort & 175 & 5.14 & 2.66 \\
\hline High EL & Medium Effort & 175 & 5.04 & 1.57 \\
\hline High EL & Low Effort & 175 & 4.82 & 1.26 \\
\hline \multicolumn{2}{|c|}{ Total for High EL } & 525 & 4.99 & 1.94 \\
\hline \multicolumn{2}{|c|}{ Total for whole sample } & 1575 & 6.30 & 1.87 \\
\hline
\end{tabular}

After examining the germane load means for each of the nine conditions, analysis of variance (ANOVA) was used to analyze the differences among and between the group means that were a part of this research. ANOVA tested if there was a statistically significant different between the high, medium and low effort groups within each of the extraneous load conditions. Table 5 shows both the low and medium extraneous load groups showed a significant difference in germane load depending on whether the participants were in the high, medium, or low effort group. However, in the high extraneous load condition, there was no statistically significant difference based on which selfregulated effort group the participant belonged to.

Table 5

ANOVA for the Different Effort Groups by Extraneous Load Group

\begin{tabular}{|l|l|l|l|l|l|l|}
\hline & & $\begin{array}{l}\text { Sum of } \\
\text { Squares }\end{array}$ & Df & $\begin{array}{l}\text { Mean } \\
\text { Square }\end{array}$ & F & Sig. \\
\hline Low EL & $\begin{array}{l}\text { Between } \\
\text { Groups }\end{array}$ & 772.988 & 2 & 386.494 & 119.008 & 0.001 \\
\hline
\end{tabular}




\begin{tabular}{|c|c|c|c|c|c|c|}
\hline & Within Groups & 1737.475 & 523 & 3.248 & & \\
\hline & Total & 2510.462 & 525 & & & \\
\hline \multirow[t]{3}{*}{ Medium EL } & $\begin{array}{l}\text { Between } \\
\text { Groups }\end{array}$ & 266.808 & 2 & 133.404 & \multirow[t]{3}{*}{$75 \cdot 342$} & \multirow[t]{3}{*}{0.001} \\
\hline & Within Groups & 952.61 & 523 & 1.771 & & \\
\hline & Total & 1219.418 & 525 & & & \\
\hline \multirow[t]{3}{*}{ High EL } & $\begin{array}{l}\text { Between } \\
\text { Groups }\end{array}$ & 27.569 & 2 & 6.785 & \multirow[t]{3}{*}{1.696} & \multirow[t]{3}{*}{0.344} \\
\hline & Within Groups & 1969.478 & 523 & 3.73 & & \\
\hline & Total & 1997.047 & 525 & & & \\
\hline
\end{tabular}

After the overall difference in each of the extraneous load conditions was established, the Scheffe test was used to examine the specific difference of germane load means between each effort group, and whether or not those differences were statistically significant. The Scheffe test is a single-step multiple comparison procedure designed to be applied to the set of estimates of all possible comparisons among means. Table 6 shows that in the low extraneous load condition, the high effort group's germane load mean was 2.894 higher than the low effort group and 1.192 higher than the medium effort group. The medium effort group's germane load mean was 1.702 higher than the low effort group. All of those differences were statistically significant.

Table 6

Scheffe Test for the Low Extraneous Load Group $(N=525)$

\begin{tabular}{|c|c|c|c|}
\hline & Low Effort & Medium Effort & High Effort \\
\hline High Effort & $2.894^{*}$ & $1.192^{*}$ & 1 \\
\hline Medium Effort & $1.702^{*}$ & 1 & \\
\hline Low Effort & 1 & & \\
\hline
\end{tabular}

* Correlation is significant at the .05 level 
The Scheffe test was also used in the medium extraneous load group to examine the specific difference of germane load means between each effort group, and whether or not those differences were statistically significant. Table 7 shows that in the medium extraneous load condition, the high effort group's germane load mean was 1.706 higher than the low effort group and 1.037 higher than the medium effort group. The medium effort group's germane load mean was 0.669 higher than the low effort group. All of those differences were statistically significant.

Table 7

Scheffe Test for the Medium EL Group $(N=525)$

\begin{tabular}{|c|c|c|c|}
\hline & Low Effort & Medium Effort & High Effort \\
\hline High Effort & $1.706^{*}$ & $1.037^{*}$ & 1 \\
\hline Medium Effort & $0.669^{*}$ & 1 & \\
\hline Low Effort & 1 & & \\
\hline
\end{tabular}

* Correlation is significant at the .05 level

Finally, the Scheffe test was used to examine the specific differences of germane load means between each effort group in the high extraneous load condition, and whether or not those differences were statistically significant. Table 8 shows that in the high extraneous load condition, the high effort group's germane load mean was 0.326 higher than the low effort group and 0.106 higher than the medium effort group. The medium effort group's germane load mean was 0.22 higher than the low effort group. Unlike in the medium and low extraneous load conditions, there was no statistical difference between the groups.

Table 8

Scheffe Test for the High EL Group ( $n=525)$

\begin{tabular}{|c|c|c|c|}
\hline & Low Effort & Medium Effort & High Effort \\
\hline High Effort & 0.326 & 0.106 & 1 \\
\hline Medium Effort & 0.22 & 1 & \\
\hline Low Effort & 1 & & \\
\hline
\end{tabular}

* Correlation is significant at the .05 level 


\section{Discussion}

The results showed that extraneous load had a negative relationship with both germane load and selfregulated effort. That is, as extraneous load reduced, both germane load and self-regulated effort increased. Furthermore, self-regulated effort had a positive relationship with germane load, with germane load increasing as self-regulated effort increased. This relationship is meaningful in that it shows that students who put in more effort are more likely to succeed. However, this study also looked at the significance of this relationship within varying extraneous load environments to see if effort can overcome unclear instruction. This study revealed that extraneous load levels varied to enough of a degree where three separate conditions could be created. As mentioned by those who participated in the qualitative interviews, the presentation of video lectures varied among different courses, which played a role in the extraneous load variation found in this study.

Accordingly, the main purpose of this study was to examine the relationships between varying levels of self-regulated effort (low, medium, high) and germane load within specific extraneous load conditions (low, medium, high). Results indicated that as extraneous load increased, the relationship between self-regulated effort and germane load weakened. Additionally, within the low and medium extraneous load conditions, germane load levels significantly differed among the three self-regulated effort groups. This shows that within those conditions, self-regulated effort had a significantly different relationship with germane load based on effort levels, with higher effort levels having higher levels of germane load. However, no significant difference was found between germane load means among the three self-regulated effort groups within the high extraneous load condition. This shows that within high extraneous load online environments, higher levels of self-regulation not only have a weaker relationship with germane load than in medium and low extraneous load environments, but that using more self-regulated effort is generally no more effective than using less self-regulated effort.

The negative correlation found between extraneous load and germane load is supported by cognitive load theory, which states that extraneous load leads to unnecessary cognitive processing, negatively affecting comprehension and ultimately germane load (Cierniak et al., 2009; De Jong, 2010; Leppink et al., 2013; Sweller et al., 1998). Experiments have shown that learners who are forced to manage extraneous load as a result of ineffective instruction generally show lower levels of comprehension (Mayer et al., 2001; Moreno et al., 2001). The negative relationship found between extraneous load and self-regulated effort is also supported by research, as use of self-regulated learning strategies within e-learning has been found to be negatively affected by extraneous load within the learning environment (Moos, 2013). Additionally, the positive relationship between self-regulated effort and germane load is not surprising, in that research has shown that self-regulation has been connected to higher levels of learning within e-learning environments (Moos, 2013; Moos \& Azevedo, 2008; Puzziferro, 2008; Shih et al., 1998).

The results of the current study involving the relationships between varying levels of self-regulated effort and germane load within specific extraneous load conditions are revealing in light of other research. Regarding the low extraneous load condition, it can be said that when clear instruction is presented, self-regulated effort is an effective strategy and that those that use it will show greater success than those that do not. Similar results regarding the difference between germane load means of the three self-regulated effort groups were found within the medium extraneous load environment; however, higher levels of self-regulated effort had a somewhat weaker relationship with germane load 
than they did within the low extraneous load condition. Thus it can be concluded that within mid-level extraneous load environments, higher levels of self-regulated effort can still have a stronger relationship with learning than lower levels of self-regulated effort. However the use of more effort may not be as successful as it would be in conditions of low extraneous load. Studies addressing similar circumstances have revealed that learning is increased through the use of higher selfregulation as a result of low extraneous load instruction (Moos \& Azevedo, 2008; Kramarski \& Mizrachi, 2006). However, these studies differ from the current study in that they did not examine learning differences between different self-regulated learning levels within different extraneous load environments. This was addressed by Gerjets et al. (2000), with results providing support for the current study in that both high and low levels of effort used for processing strategies were examined within a low extraneous load e-learning environment, and that low-effort learners were outperformed by high-effort learners.

The current study's results from the high extraneous load condition are much more significant in terms of exposing the exceptional negative influence that high levels of extraneous load have on the learning experience. This is evident within that condition by the fact that higher levels of selfregulated effort had no relationship with germane load, unlike the low and medium extraneous load conditions. Similar germane load means were found among the three self-regulated effort groups, showing that high extraneous load was so significant that high levels of self-regulated effort were just as ineffective as lower levels of self-regulated effort in overcoming poor instruction. Moos (2013) showed that high extraneous load within an e-learning environment decreases effort, leading to lower levels of learning. However, Moos (2013) did not look at the use of high self-regulated learning strategies within high extraneous load environments to see if self-regulation can be effective in overcoming poor instruction. To examine this phenomenon, Gerjets et al. (2000) showed that students displaying high levels of effort within a high extraneous load environment showed no difference in performance compared to students in a low extraneous load condition, effectively overcoming poor instruction.

The current study makes an important contribution to the field of e-learning in that it brings to light that there are some situations where student effort will not be effective in overcoming poor instruction. Some research shows that certain types of learners show higher levels of performance, regardless of the instructional situation. Specifically, it is generally accepted that motivated students who display high levels of effort can overcome obstacles faced within the learning environment and ultimately succeed in their coursework. Research has suggested, for example, that effort involved in selfregulation can overcome the delivery of incomprehensible material, inadequate study conditions, and confusing instruction (Gerjets et al., 2000; Leutner \& Sumfleth, 2009; Moos, 2011; Zimmerman, 1990). Gerjets et al. (2000) for example, claim that students who use more effort can overcome poor instruction unlike the current study in which high effort of levels could not overcome poor instruction. The fact that in the present study effort was unsuccessful in overcoming the perceived unclear instruction can be explained by the processes associated with extraneous load as part of cognitive load theory. Unlike germane load, extraneous load is associated with the effort that is either unnecessary or irrelevant to learning (Cierniak et al., 2009; Leppink et al., 2013; Schmeck et al., 2015). The effort that goes into dealing with confusing instruction may ultimately create extraneous processing, as students are using cognitive effort to make sense of unclear instruction, which does not contribute to learning. Self-regulated effort measurements look at whether students who face obstacles in the learning 
environment persist by exerting effort to complete their work. This may be effective when facing complex material that is well taught. This is because students perceive that their effort leads to learning. However, when students deal with extraneous processing through unclear explanation of the content, more effort may be ineffective because it is not directed towards meaningful learning.

\section{Conclusion}

Results from this study show that the effort put forth by students to overcome challenging situations may not always lead to successful learning. However, the results of this study also revealed that when students face lower levels of extraneous load within an e-learning environment, higher levels of selfregulated effort have a positive relationship with learning. The implications of this research are useful for both instructors and learners within multimedia e-learning environments. While e-learning instructors need to present instruction in a way that avoids the creation of extraneous processing by students, e-learning students should self-regulate their effort to gain a better understanding of the content. Based on the findings of the current study, however, it is important to note that performance gains are more likely to occur within e-learning when both of those conditions are simultaneously met.

There are several ways in which instructors can avoid the delivery of extraneous information and allow students to apply effort toward meaningful learning. For example, instructors should consider presenting students with worked examples to problems within e-learning environments, which clears up instructional confusion through scaffolding of procedural steps (Gergets, 2000). Segmenting is an in instructional technique that allows for a controlled pace of learning because it presents the content in smaller chunks at a single time, allowing students to digest the information before going on to the next level of explanation (Mayer \& Moreno, 2003). Additionally, students can watch each segment within a video lecture multiple times and pause the video in order to think about the content before moving on to the next segment. Ultimately, it clarifies instruction by avoiding unclear content that may occur if information is presented all at once. Teaching strategies such as these not only contribute to clear instructional delivery, but they can also increase chances of strategic effort. This is according to research that claims students are more likely to use specific strategies when levels of extraneous load are minimized (Moos, 2013; Pass et al., 2005). It is important to note that presenting the content clearly is by no means a panacea, and that students who use lower levels of effort will still exist. But if the content is clearly presented, effort that is directed to such content should lead to higher levels of understanding due to the fact that such explanation contributes to meaningful learning.

Although the results of this study contribute to the discourse of online learning research through evidence that student effort may not always overcome poor instruction, there are some limitations. This study was based on survey responses of subjective measurements involving cognitive load and self-regulated effort. The results can be strengthened by examining the same relationships within a controlled experimental environment. Specifically, creating online instruction containing various levels of extraneous load and having students modify their use of self-regulated learning strategies within specific extraneous load environments, may further support these results. Given that, the results of the current study build on what previous research has done regarding the conceptualization of complex relationships between cognitive load and self-regulated learning within multimedia elearning environments. 


\section{References}

Chang, C.-C., \& Yang, F.-Y. (2010). Exploring the cognitive loads of high-school students as they learn concepts in web-based environments. Computers \& Education, 55(2), 673-680.

Cierniak, G., Scheiter, K., \& Gerjets, P. (2009). Explaining the split-attention effect: is the reduction of extraneous cognitive load accompanied by an increase in germane cognitive load? Computers in Human Behavior, 25, 315-324.

Dabbagh, N., \& Kitsantas, A. (2012). Personal Learning Environments, social media, and selfregulated learning: A natural formula for connecting formal and informal learning. The Internet and Higher Education, 15(1), 3-8.

De Jong, T. (2010). Cognitive load theory, educational research, and instructional design: some food for thought. Instructional Science, 38(2), 105-134.

Duncan, T. G., \& McKeachie, W. J. (2005). The making of the motivated strategies for learning questionnaire. Educational Psychologist, 4O(2), 117-128.

Ergul, H. (2004). Relationship between student characteristics and academic achievement in distance education and application on students of Anadolu University. Turkish Online Journal of Distance Education, 5(2), 81-90.

Gerjets, P., Scheiter, K., \& Tack, W. H. (2000). Resource-adaptive selection of strategies in learning from worked-out examples. In L. R. Gleitman \& A. K. Joshi (Eds.), Proceedings of the 22nd Annual Conference of the Cognitive Science Society (pp. 166-171). Mahwah, NJ: Erlbaum.

Gerjets, P. \& Scheiter, K. (2003). Goal configurations and processing strategies as moderators between instructional design and cognitive load: Evidence from hypertext-based instruction. Educational Psychologist, 38(1), 33-41.

Han, S. (2012). A study on the development of a course evaluation tool for Cyber University Consortium in Korea. In Kim, T., Mohammed, S., Ramos, C., Abawajy, J., Kang, B., \& Slezak D. (Eds.), Computer applications for web, human computer interaction, signal and image processing, and pattern recognition (pp. 331-337).

Homer, B. D., Plass, J. L., \& Blake, L. (2008). The effects of video on cognitive load and social presence in multimedia-learning. Computers in Human Behavior, 24(3), 786-797.

Jung, I. (2000). Korea: Virtual university trial project. TechKnowLogia, 29-31.

Jung, I. \& Rha, I. (2001). A virtual university trial project: Its impact on higher education in South Korea. Innovations in Education and Teaching International, 38(1), 31-41. 
Kalyuga, S., Chandler, P., \& Sweller, J. (1999). Managing split-attention and redundancy in multimedia instruction. Applied Cognitive Psychology, 13, 351-371.

Kirschner, P. A. (2002). Cognitive load theory: Implications of cognitive load theory on the design of learning. Learning and Instruction, 12, 1-10.

Kobayashi, T. \& Kim, Y. (2010). The status of cyber education in Korean higher learning: The potential for East Asian linkage in higher education. The Open University of Japan Repository. Accessed August 25 ${ }^{\text {th }}$, 2016, from http://id.nii.ac.jp/1146/00003502/

Komarraju, M., \& Nadler, D. (2013). Self-efficacy and academic achievement: Why do implicit beliefs, goals, and effort regulation matter? Learning and Individual Differences, 25, 67-72.

Kramarski, B. \& Mizrachi, N. (2006). Online discussion and self-regulated learning: effects of instructional methods on mathematical literacy. Journal of Educational Research, 99(4), 218-230.

Leppink, J., Paas, F., Van der Vleuten, C. P., Van Gog, T., \& Van Merriënboer, J. J. (2013). Development of an instrument for measuring different types of cognitive load. Behavior Research Methods, 45(4), 1058-1072.

Leutner, D., Leopold, C., \& Sumfleth, E. (2009). Cognitive load and science text comprehension: Effects of drawing and mentally imagining text content. Computers in Human Behavior, 25(2), 284-289.

Mayer, R. E. (2003). Elements of a science of e-learning. Journal of Educational Computing Research, 29(3), 297-313.

Mayer, R. E. (2014). Cognitive theory of multimedia learning. The Cambridge handbook of multimedia learning, 43-71.

Mayer, R. E., Heiser, J., \& Lonn, S. (2001). Cognitive constraints on multimedia learning: When presenting more material results in less understanding. Journal of Educational Psychology, 93(1), 187-198. http://doi.org/10.1037/0022-0663.93.1.187

Mayer, R. E., \& Moreno, R. (2003). Nine ways to reduce cognitive load in multimedia learning. Educational Psychologist, 38(1), 43-52.

McManus, T. (2000). Individualizing instruction in a Web-based hypermedia learning environment: Nonlinearity, advanced organizers, and self-regulated learners. Journal of Interactive Learning Environments, 11, 219-251.

Moos, D. (2013). Examining hypermedia learning: The role of cognitive load and self-regulated learning. Journal of Educational Multimedia and Hypermedia, 22(1), 39-61.

Moos, D. C., \& Azevedo, R. (2008). Monitoring, planning, and self-efficacy during learning with hypermedia: The impact of conceptual scaffolds. Computers in Human Behavior, 24(4), 
$1686-1706$.

Moreno, R., Mayer, R. E., Spires, H. A., \& Lester, J. C. (2001). The case for social agency in computerbased teaching: Do students learn more deeply when they interact with animated pedagogical agents? Cognition and instruction, 19(2), 177-213.

Nie, Y., Lau, S., \& Liau, A. K. (2011). Role of academic self-efficacy in moderating the relation between task importance and test anxiety. Learning and Individual Differences, 21(6), 736-741.

Paas, F., Tuovinen, J., van Merriënboer, J. J. G., \& Darabi, A. (2005). A motivational perspective on the relation between mental effort and performance: Optimizing learner involvement in instruction. Educational Technology, Research and Development, 53, 25-34.

Perry, N.E., Phillips, L., \& Hutchinson, L.R. (2006). Preparing student teachers to support for selfregulated learning. Elementary School Journal, 106, 237-254.

Pintrich, P., Smith, D. A. F., Garcia, T., \&.McKeachie, W. J. (1991). A manual for the use of the motivated strategies for learning questionnaire (MSLQ) (Technical Report No. 91-B-004) Ann Arbor: University of Michigan National Center for Research to Improve Postsecondary Teaching and Learning.

Puzziferro, M. (2008). Online technologies self-efficacy and self-regulated learning as predictors of final grade and satisfaction in college-level online courses. The American Journal of Distance Education, 22(2), 72-89.

Saw, A. T. (2011). Learner Control, Expertise, and Self-Regulation: Implications for Web-Based Statistics Tutorials (Doctoral dissertation, Claremont Graduate University).

Schmeck, A., Opfermann, M., van Gog, T., Paas, F., \& Leutner, D. (2015). Measuring cognitive load with subjective rating scales during problem solving: differences between immediate and delayed ratings. Instructional Science, 43(1), 93-114.

Shadiev, R., Hwang, W. Y., Huang, Y. M., \& Liu, T. Y. (2015). The impact of supported and annotated mobile learning on achievement and cognitive load. Journal of Educational Technology \& Society, 18(4), 53-69.

Shih, C., Ingebritsen, T., Pleasants, J., Flickinger, K., \& Brown, G. 1998. Learning strategies and other factors influencing achievement via Web courses. Proceedings of the 14th Annual Conference on Distance Teaching and Learning (359-363). Madison, WI. (ED 422876)

Simon, R. A., Aulls, M. W., Dedic, H., Hubbard, K., \& Hall, N. C. (2015). Exploring student persistence in STEM programs: A motivational model. Canadian Journal of Education, 38(1), 1-27.

Suh, S., \& Kim, S. (2013). Study on policy for an entrance quota of cyber universities. Seoul, Korea: Korea Educational Information and Research Service.

Sweller, J. (2005). Implications of cognitive load theory for multimedia learning. In R. E. Mayer (Ed.), 
The Cambridge handbook of multimedia learning (pp. 19-30). New York: Cambridge University Press.

Sweller, J., \& Chandler, P. (1994). Why some material is difficult to learn. Cognition and Instruction, 12, 185-233.

Sweller, J., Van Merriënboer, J. J. G., \& Paas, F. (1998). Cognitive architecture and instructional design. Educational Psychology Review, 10, 251-296.

Wang, T. H. (2011). Developing web-based assessment strategies for facilitating junior high school students to perform self-regulated learning in an e-Learning environment. Computers \& Education, 57(2), 1801-1812.

Winters, F. I., Greene, J. A., \& Costich, C. M. (2008). Self-regulation of learning within computerbased learning environments: A critical analysis. Educational Psychology Review, 2O(4), 429-444.

Zimmerman, B. J. (1990). Self-regulated learning and academic achievement: An overview. Educational Psychologist, 25(1), 3-17.

Zimmerman, B., J (2008). Investigating self-regulation and motivation: Historical background, methodological developments and future prospects. American Educational Research Journal, 45(1), 166-183.

Zimmerman, B. J., \& Pons, M. M. (1986). Development of a structured interview for assessing student use of self-regulated learning strategies. American Educational Research Journal, 23(4), 614628.

\section{Athabasca \\ University}

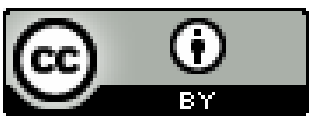

